Cinémas

Revue d'études cinématographiques

Journal of Film Studies

\title{
Composition en profondeur, mobilité et montage dans Cabiria (Pastrone, 1914)
}

\section{Elena Dagrada, André Gaudreault et Tom Gunning}

Volume 10, numéro 1, automne 1999

Cinélekta 3

URI : https://id.erudit.org/iderudit/024803ar

DOI : https://doi.org/10.7202/024803ar

Aller au sommaire du numéro

Éditeur(s)

Cinémas

ISSN

1181-6945 (imprimé)

1705-6500 (numérique)

Découvrir la revue

Citer cet article

Dagrada, E., Gaudreault, A. \& Gunning, T. (1999). Composition en profondeur, mobilité et montage dans Cabiria (Pastrone, 1914). Cinémas, 10(1), 55-78.

https://doi.org/10.7202/024803ar
Résumé de l'article

Une étude comparative du film Cabiria et de ses chutes (prises de vues exclues de son montage définitif) permet de montrer comment la mise en scène de ce film italien, sorti en 1914, est fondée sur une forme de composition spatiale en profondeur. D'où la tendance notable d'y éviter la fragmentation de l'espace via le montage, comme pour en respecter l'intégrité et mettre en valeur son aspect tridimensionnel. À l'image de la majorité des films européens de la première moitié des années dix, Cabiria réalise ainsi un idéal de représentation spatiale tridimensionnelle « à circularité limitée » qui, tout en permettant au spectateur de se rapprocher de la scène, ne situe jamais l'instance spectatorielle au centre de l'action, un idéal de représentation spatiale au sein de laquelle la mobilité de l'espace scénique est obtenue autrement que par le changement de plan. 


\title{
Composition en profondeur, mobilité et montage dans Cabiria (Pastrone, 1914) ${ }^{1}$
}

\author{
Elena Dagrada, André Gaudreault \\ et Tom Gunning
}

\begin{abstract}
RÉSUMÉ
Une étude comparative du film Cabiria et de ses chutes (prises de vues exclues de son montage définitif) permet de montrer comment la mise en scène de ce film italien, sorti en 1914, est fondée sur une forme de composition spatiale en profondeur. D'où la tendance notable d'y éviter la fragmentation de l'espace via le montage, comme pour en respecter l'intégrité et mettre en valeur son aspect tridimensionnel. $\grave{A}$ l'image de la majorité des films européens de la première moitié des années dix, Cabiria réalise ainsi un idéal de représentation spatiale tridimensionnelle "à circularité limitée " qui, tout en permettant au spectateur de se rapprocher de la scène, ne situe jamais l'instance spectatorielle au centre de l'action, un idéal de représentation spatiale au sein de laquelle la mobilité de l'espace scénique est obtenue autrement que par le changement de plan.
\end{abstract}

\section{ABSTRACT}

Through the comparative study of Cabiria and the footage excluded from the final cut, this essay demonstrates that this Italian film, released in 1914, follows a project of "in-depth spatial composition" in an attempt both to avoid the fragmentation of the represented space caused by editing and to respect its integrity through an emphasis on its tridimensional aspect. Like the majority of European films of the first half of the 1910s, Cabiria thus realizes an ideal of 
tridimensional spatial representation "with limited circularity" (which, while it enables the spectator to get closer to the scene, never situates it at the centre of the action). The dominant feature of this type of spatial representation is the mobility of the scenic space, which is different from the one created by the succession of shots.

Le texte qui suit est le résultat d'une recherche menée à trois ${ }^{2}$, sur la base d'une étude comparative de Cabiria (Itala Film, 1914) et des prises de vues ayant été exclues de son montage définitif. Retrouvées au Museo nazionale del cinema de Turin, à l'occasion de la récente opération de restauration qui a redonné au film de Pastrone une longueur de 3343 mètres ${ }^{3}$, ces trois heures de chutes ont été, pour nous, un objet d'étude de nature plus spéculative que documentaire, plus réflexive qu'empirique. Notre approche a, en effet, été essentiellement analytique et textuelle. Il ne s'agissait pas, pour nous, de mener une enquête sur les péripéties de la conservation de ces chutes ni de nous pencher sur le contexte de production de Cabiria ou sur les conditions de sa réception. En cours de route, cette recherche, qui devait porter strictement sur les rapports entre la version finale de Cabiria et les chutes du film, a dévié de son itinéraire principal pour emprunter une autre voie, dont l'importance est loin d'être secondaire. L'étude de ces chutes nous a en effet donné l'extraordinaire opportunité d'entrer dans le laboratoire de mise en scène d'un "auteur " italien de la première moitié des années dix et nous a poussés à nous demander quel projet esthétique pouvait être à la base d'un film aussi complexe que Cabiria.

La légende veut que Giovanni Pastrone, directeur artistique et superviseur de la production à l'Itala Film, ait personnellement édicté le règlement, en vigueur à l'Itala, interdisant de tourner une deuxième prise d'une scène, sauf si la chose s'avérait "absolument nécessaire ${ }^{4}$ ". À en juger par la quantité et la qualité des chutes de Cabiria, on doit bien conclure que Pastrone ne respecta pas son propre règlement, du moins dans le cas de ce film. On pourrait y voir un signe du zèle qui fut déployé pour la production de Cabiria, mais il s'agit également d'une trace, par 
ailleurs très éloquente, du véritable travail de recherche mis en œuvre pour la réalisation de ce film.

Autant le dire tout de suite, ce dont l'abondant matériel que représentent les chutes de Cabiria témoigne, c'est que le recours aux prises multiples n'a presque jamais été provoqué par des raisons d'absolue nécessité. Bien sûr, parmi les plans exclus, il s'en trouve qui, fort semblables à ceux choisis pour le montage définitif, paraissent avoir été repris essentiellement pour des raisons d'ordre technique. On trouve en effet, dans le lot des chutes, des cas de défection du "filmographique" (problème d'éclairage, mauvaise exposition, etc.) ou du "profilmique" (colombe qui ne vole pas, chameau qui ne suit pas la trajectoire prévue, acteur qui trébuche ${ }^{5}$...). Mais ce sont là des exceptions. Et, encore, montrent-elles que Pastrone s'est parfois servi, de façon exemplaire, de pareille erreur pour expérimenter diverses alternatives de cadrage (ou autre chose encore ${ }^{6}$ ).

Notre recherche nous a donc permis de constater que, en dehors de ces exceptions, la plus grande partie des plans exclus ont été tournés dans le but d'expérimenter différentes mises en scène d'une même action, de vérifier l'efficacité d'une composition spatiale en profondeur, d'accélérer ou de ralentir une action ou de se donner le choix entre un plan fixe et un carrello (travelling). Ou encore, plus simplement, afin de se constituer une banque d' "options" (de nombreux plans des chutes n'apparaissent tout simplement pas dans le film, remplacés qu'ils ont été par une tout autre solution de mise en scène).

L'examen de l'ensemble des chutes de Cabiria a d'ailleurs pu nous permettre de constater que ce film était le fruit d'un projet esthétique bien circonscrit. Aussi n'avons-nous jamais considéré ces chutes comme un ensemble de scories dues à des "erreurs", comme du matériel rejeté parce que, présumément, "mal fait». Nous avons évité d'analyser ces chutes comme un répertoire dans lequel il nous serait loisible de retracer d'éventuelles imperfections à interpréter comme la cause de leur exclusion. Au contraire, nous les avons constamment envisagées comme une trace, très précieuse, du fait que pendant la première moitié des années dix, en Italie, on n'hésitait pas à tourner plusieurs fois la même scène, le même plan, afin d'expérimenter diverses possibilités esthétiques. 
En confrontant le film Cabiria avec ses chutes, il nous a semblé que, dans son travail sur la mise en scène, Pastrone avait nettement concentré ses efforts sur la dimension spatiale. Bien sûr, dans Cabiria, le traitement du temps revêt, lui aussi, son importance, à la fois en termes de rythme et parce que le temps de la narration y est toujours linéaire et chronologique? Néanmoins, et en vertu notamment de cette linéarité, c'est dans l'analyse spatiale que le travail de recherche dans la mise en scène, dans Cabiria, nous a paru particulièrement riche et ce, pour deux raisons.

Disons, d'entrée de jeu, que Cabiria est un film dans lequel on trouve pratiquement toutes les figures de montage élaborées jusque-là (champ/contrechamp, raccord dans l'axe, raccord de direction, plans subjectifs et raccords sur un regard, alternance d'espaces contigus, etc.). Il s'agit donc d'un film dans lequel le montage (au sens large) est loin d'être absent, ce que l'analyse des chutes corrobore d'ailleurs. Mais, en même temps, le type de mise en scène qui y prévaut relève de ce que nous appellerons une "composition spatiale en profondeur ${ }^{8}$ ", parce qu'elle tend à éviter la fragmentation de l'espace représenté et à le mettre en scène, autant que possible, dans son intégrité. Cette "composition spatiale en profondeur" résulte en fait d'une volonté de représenter un espace en mettant en valeur son aspect «tridimensionnel " (à ne pas confondre avec la tendance à la mise en scène d'un espace filmique plus proprement "circulaire ", déjà à l'œuvre dans le cinéma américain de l'époque). Elle se présente comme une alternative au montage entendu comme «fragmentation » d'un même espace en plusieurs plans, s'articulant davantage comme une «mise en cadre» en profondeur que comme une "mise en chaîne" de points de vue divers sur un même décor, ce qui n'entre pas en contradiction avec la coprésence, dans le film, des principales figures de montage. Le montage est en effet loin d'être absent d'un film comme Cabiria, bien qu'il soit très rarement au service de la fragmentation spatiale d'un même lieu.

La figure de montage vraisemblablement la plus fréquente dans le film est même l'une des figures par excellence du montage: l'alternance entre l'extérieur et l'intérieur d'un immeuble 
ou d'une pièce. Dans Cabiria, en effet, on passe souvent de l'intérieur à l'extérieur du temple de Moloch, de l'auberge de Bodastorèt ou du palais royal de Carthage. Létude des chutes montre d'ailleurs que cette figure a été réalisée, à l'époque, à peu près comme on le fait aujourd'hui, c'est-à-dire en tournant plusieurs plans en continuité et en les fragmentant au montage, de façon à créer une alternance intérieur/extérieur.

Nous en trouvons un exemple éloquent dans le second épisode, alors que Maciste et Fulvio se rendent pour la première fois chez l'aubergiste. Dans les chutes, nous disposons pour cette scène de deux plans différents, relativement longs (et d'un troisième plus bref, à peu près semblable à la fin du second). Le premier plan représente l'extérieur de l'auberge: Maciste et Fulvio s'avancent et pénètrent dans l'auberge. Au même moment, un client sort de l'auberge et un carrello latéral vers la gauche l'accompagne. Un second plan représente l'intérieur de l'auberge. Fulvio et Maciste entrent dans le champ par la gauche et passent entre les tables, en avançant vers la droite. Puis, ils s'assoient et se font servir. Suit alors un carrello avant, extrêmement lent, qui s'approche de Maciste, occupé à boire. Or, dans le film, les deux prises qui ont été retenues (et qui sont à peu près équivalentes à celles que nous venons de décrire) sont divisées en quatre plans, qui alternent point de vue extérieur et point de vue intérieur. Plan $n^{\circ} 1$, extérieur, Maciste et Fulvio s'avancent et entrent; plan $\mathrm{n}^{\circ} 2$, dans l'auberge, Maciste et Fulvio entrent dans le champ et s'assoient; plan $\mathrm{n}^{\circ} 3$, à nouveau à l'extérieur, le client sort, alors que la caméra le suit; plan $\mathrm{n}^{\circ} 4$, retour à l'intérieur, un carrello avant s'approche de la table où sont assis Fulvio et Maciste.

Nous pourrions citer d'autres exemples analogues où, en plus, l'alternance se produit à la faveur d'un renversement de $180^{\circ}$ dans l'angle de prise de vues. Ainsi en est-il, par exemple, de ce segment du second épisode dans lequel Maciste contraint l'aubergiste à se montrer à la fenêtre (d'abord située dans l'auberge, la caméra montre ce dernier de dos, pour se retrouver, quelques instants plus tard, à l'extérieur de l'auberge, nous le montrant en train de passer la tête par la fenêtre).

Il n'est par ailleurs qu'un seul moment dans le film où l'on assiste à l'analyse d'un même espace (à la faveur, incidemment, 
d'un renversement de l'angle de prise de vues par $\left.180^{\circ}\right)^{\circ}$. Il s'agit de cette scène du second épisode qui représente la rencontre entre Croessa, Fulvio et Maciste, un moment véritablement crucial dans l'intrigue, puisque c'est celui où les deux histoires, celle de Cabiria et celle de Fulvio/Maciste, se croisent pour la première fois. C'est peut-être pour cela que Pastrone fait, ici, l'essai de deux mises en scène assez différentes l'une de l'autre, allant même jusqu'à tourner la même action dans deux décors différents (tout en conservant le même type de fragmentation spatiale).

On trouve dans les chutes de nombreuses prises tournées dans le décor ayant été éliminé. Un premier plan (deux prises) représente une terrasse qui surplombe la mer. Croessa s'avance en direction d'une bitte d'amarrage, derrière laquelle elle s'affaisse (photo $\mathrm{n}^{0} 1$ ). Un deuxième plan (deux prises, également) montre Croessa, à partir d'un angle opposé. Elle est appuyée contre la bitte, tandis que Fulvio et Maciste avancent en sa direction (photo $\mathrm{n}^{\circ} 2$ ). Un troisième plan (deux prises, ici aussi) renverse à nouveau l'angle de prise de vues. Croessa implore Fulvio et Maciste de sauver Cabiria (photo $n^{\circ} 3$ ).

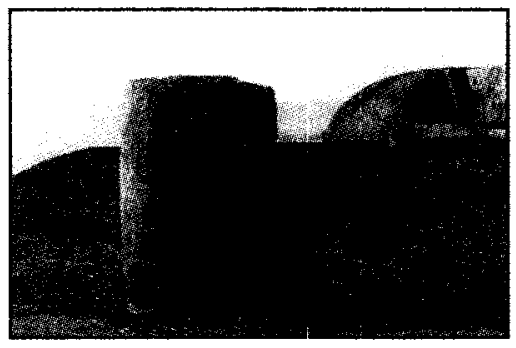

Photo $\mathrm{n}^{0} 1$

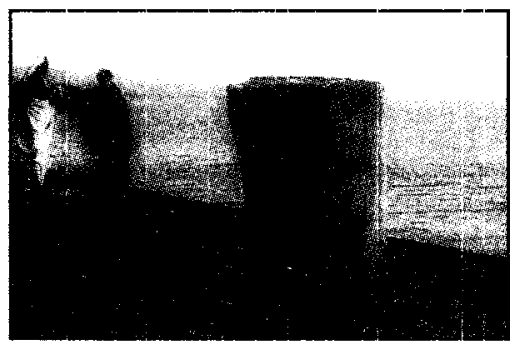

Photo $\mathrm{n}^{\circ} 2$

Cabiria (Giovanni Pastrone, 1914)

Dans le montage définitif, Pastrone ne retient aucune de ces prises. Il adopte en effet l'autre décor, qui campe l'action sur les remparts de la ville. Sur le plan structurel, il privilégie un découpage du même type que celui que l'on vient de décrire, avec toutefois d'énormes différences en ce qui a trait, du moins, au nombre de plans et à l'échelle des plans. Un premier plan 


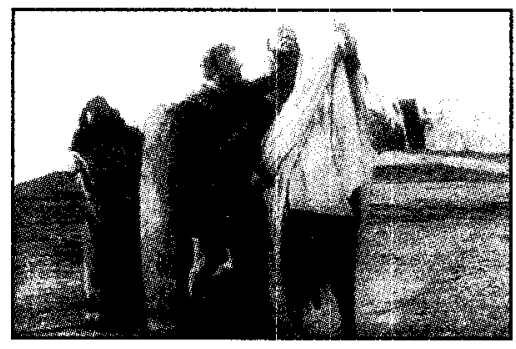

Photo $\mathbf{n}^{0} 3$

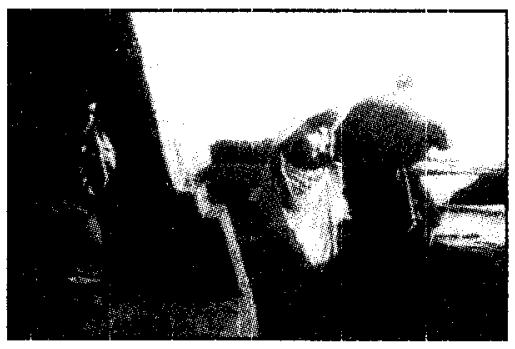

Photo $n^{0} 4$

Cabiria (Giovanni Pastrone, 1914)

montre Croessa qui longe les remparts de Carthage surplombant la mer. Elle entend au loin Fulvio et Maciste qui s'approchent (photo $\mathrm{n}^{\circ} 4$ ). Un deuxième plan fait basculer l'angle de prise de vues, pour montrer Croessa qui s'approche de Fulvio et Maciste (photo $n^{\circ} 5$ ). Un carton s'interpose et, en lieu et place d'un renversement de $180^{\circ}$ (comme dans la version rejetée), le montage présente un raccord dans l'axe, en plan rapproché $(c u t-i n)$. On ne trouve rien d'équivalent dans les chutes (photo $\mathrm{n}^{\circ} 6$ ). Ce plan est finalement suivi d'un retour au cadrage du plan précédant l'intertitre, par un raccord dans l'axe, en recul (photo $n^{\circ} 7$ ). Pour le premier "raccord" dans l'axe - les guillemets sont là parce que, vu l'interposition du carton, il n'y a pas vraiment raccord - Pastrone avait le choix de deux autres prises, qui se trouvent dans les chutes: une première, en tout point semblable à celle qui fut retenue, et une deuxième, représentant une sorte de contrechamp, dans lequel Maciste se trouve

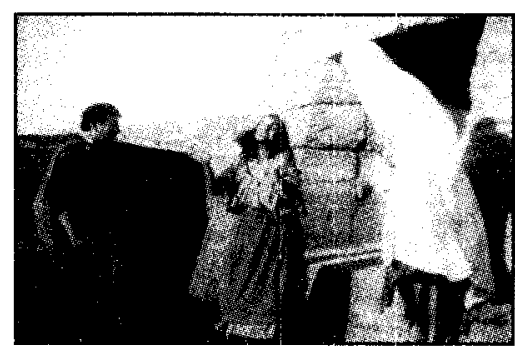

Photo $\mathbf{n}^{\circ} 5$

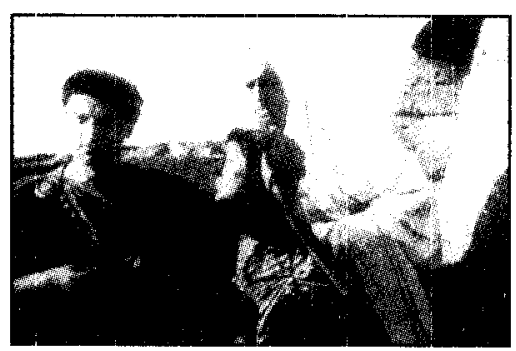

Photo $n^{0} 6$

Cabiria (Giovanni Pastrone, 1914) 
toutefois toujours à droite dans le décor (photo $\mathrm{n}^{\circ} 8$ ). Ce dernier plan a peut-être justement été exclu en raison du mauvais positionnement de Maciste, mais il a de toute façon été conçu et réalisé comme un contrechamp du plan précédent.

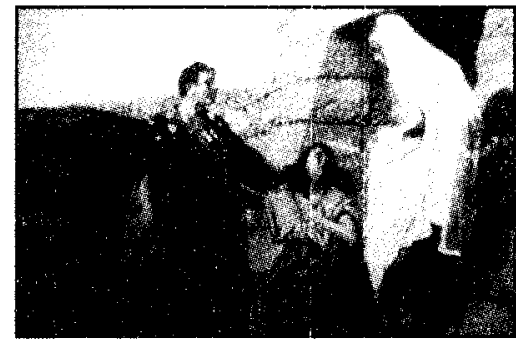

Photo $\mathbf{n}^{\circ} 7$

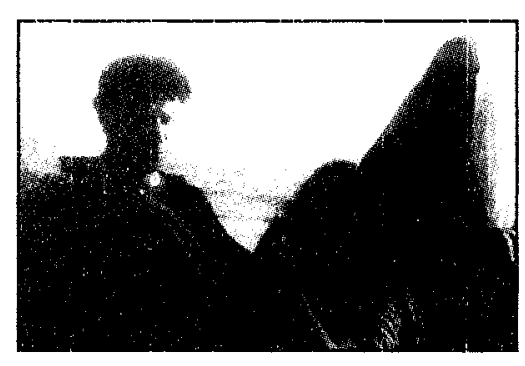

Photo $\mathrm{n}^{\circ} 8$

Cabiria (Giovanni Pastrone, 1914)

Une première remarque s'impose ici: la rencontre des deux histoires (celle de Croessa d'une part, celle de Fulvio et Maciste de l'autre), leur "entrecroisement", s'incarne tellement bien dans un "croisement» des angles de prise de vues qu'au moment du tournage, Pastrone prévoit un renversement dans l'angle de prise de vues, par un raccord de $180^{\circ}$. Sans savoir encore dans quel lieu cette scène se déroulera dans la version définitive, Pastrone sait déjà qu'il renversera l'angle de prise de vues puisque, aussi bien, il le fait dans les deux sites de tournage. Une seconde réflexion s'impose cependant à nous car, bien que ce passage témoigne d'une grande aisance dans la réalisation de cette figure de montage, même à l'intérieur d'un même décor, il faut noter qu'il s'agit là d'un cas isolé et fortement motivé.

Dans le reste du film, en effet — nous l'avons déjà dit —, il n'y a aucun autre exemple du genre. Même dans le cas des champ/contrechamp entre des personnages qui discutent assez rares par ailleurs - , ce sont toujours des espaces distincts qui alternent; distincts, bien que nécessairement contigus. Ainsi, par exemple, dans le cinquième épisode, au moment où Ellissa donne à boire aux prisonniers à travers les barreaux d'une fenêtre (photos $n^{\circ} 9$ et 10 ). Ainsi, encore, de cette autre scène qui montre les murs de Cirta assaillis par les soldats romains, au 
cinquième épisode, et qui se déroule entièrement en un lieu unique. Il est vrai que l'action y est dynamisée par la succession des 11 plans qui la composent, mais la caméra reste effectivement toujours "en deçà " des murs, extérieure à ceux-ci, et les variations d'angles de prise de vues et d'échelle dont cette scène témoigne sont pour le moins minimales (elle ne comporte que des plans d'ensemble et des plans moyens).

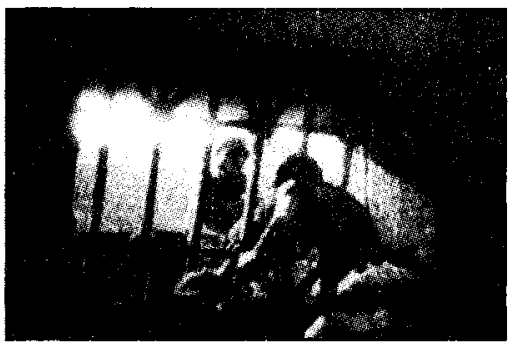

Photo $\mathbf{n}^{\circ} 9$

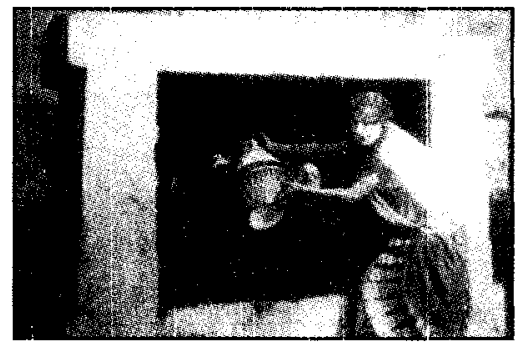

Photo $\mathrm{n}^{\circ} 10$

\section{Cabiria (Giovanni Pastrone, 1914)}

On trouve certes, dans Cabiria, les principales figures de montage élaborées jusque-là ; l'analyse des chutes peut bien, qui plus est, montrer leur caractère concerté et planifié, il n'en reste pas moins que ces figures n'en sont pas pour autant très fréquentes. Elles semblent même n'intervenir que sur la base d'une sorte de principe - que l'on pourrait définir comme une forme de "circularité limitée »-, qui réduit à l'essentiel l'utilisation du montage entendu comme fragmentation d'un même espace. Ce qui ne va pas, bien sûr, sans limiter la "circulation» du point de vue à l'intérieur d'un espace donné. Ledit principe semble, qui plus est, ne permettre le recours à la fragmentation spatiale que dans les situations où celle-ci apparaît absolument nécessaire. À l'image, du reste, de ce qui prévaut dans la majorité des films européens de la période - c'est-à-dire de la première moitié des années dix -, à l'intérieur desquels on donne préséance à la composition spatiale en profondeur sur la fragmentation spatiale via le montage.

Ainsi, dans Cabiria, le montage intervient-il plus volontiers, nous l'avons vu, pour alterner des espaces différents et 
pratiquement voisins (l'intérieur et l'extérieur d'un édifice, deux pièces différentes d'un palais, etc.) que pour fragmenter l'espace dans lequel se déroule l'action. Quand on fragmente l'espace, c'est soit pour des raisons dramaturgiques bien particulières (comme la rencontre entre Croessa, Fulvio et Maciste; ou lors de l'attaque des murs de Cirta), soit pour mettre en évidence ce qui ne pourrait l'être autrement. Un peu comme la chose se produit, selon David Bordwell, dans certains films de Louis Feuillade, qui :

[...] stubbornly adheres to a precept that he will
provide a close-up only if something is not fully visible
in the long shot because it is tiny (printed matter, a
cameo), peripheral (a hand under a bed), or blocked
from view. But this way of putting the matter makes
cutting seem only a last resort. In fact, cuts can also
reaffirm depth more actively by relocating scenic
elements in varied spatial relations (p. 22).

Cette règle du recours au montage comme ultime ressort, chez Feuillade, s'applique aussi à Pastrone qui, très souvent, semble lui aussi ne faire appel à la fragmentation de l'espace que lorsque les circonstances l'y obligent. Ainsi ne fait-il, dans Cabiria, que très rarement des "détails" (c'est-à-dire, des gros plans sur un objet ou la partie d'un corps) ${ }^{10}$ - en fait, il n'y en a que deux dans tout le film: celui sur la bague que Croessa examine, en plan subjectif, au début du second épisode, et celui sur la main devant le feu, durant le sacrifice à Moloch. Pastrone n'opère également que de très rares raccords dans l'axe sur ses personnages. Il ne le fait, en tout cas, que si la chose s'avère absolument nécessaire. En pareille circonstance, il n'avancera d'ailleurs jamais sa caméra trop près d'eux, son but n'étant, finalement, que de rendre accessible au regard du spectateur ce qui, autrement, ne se verrait pas suffisamment bien. Ce principe est confirmé par l'étude des autres cas de raccords dans l'axe, qui ne sont pas très nombreux dans le film, et qui reviennent dans des circonstances analogues.

Prenons l'exemple du sacrifice dans le temple de Moloch. La fragmentation de l'espace, par le montage, est un procédé bien évidemment incontournable pour rendre compte, dans son 
ensemble, d'un lieu et d'un décor aussi grandiose que celui-là. Dans cette scène, Pastrone utilise bien, de temps à autre, le carrello pour donner une vue de l'ensemble du décor, mais le recours au raccord dans l'axe - en général vers l'avant, parfois vers l'arrière - était, somme toute, inévitable. C'était en effet la solution qui s'imposait, si l'on voulait que le spectateur puisse observer ce qui se passe au loin, en arrière-plan (en pareil cas, un carrello eût pu être préférable, mais il aurait été plus ou moins praticable, tant en raison de l'importance de la foule des figurants qu'en raison de la distribution sur la verticale des divers niveaux de l'action représentée).

Ainsi, en outre, de cet autre raccord, presque dans l'axe, qui intervient au second épisode et qui nous montre l'aubergiste à la fenêtre, après le plan d'ensemble dont il a été question plus haut. Le personnage occupe d'abord une position périphérique dans l'image, dans la partie supérieure du cadre; le plan qui suit nous le montre de plus près, tandis qu'il tente d'expliquer aux poursuivants de Maciste qu'il n'a vu personne.

L'hypothèse voulant que, dans Cabiria, Pastrone n'ait eu recours au raccord dans l'axe qu'avec parcimonie se voit confirmée par l'étude des chutes. On trouve en effet, dans le lot des prises rejetées, quelques cas de cette figure. Même s'ils ne comportent aucune faute évidente, les plans en question n'ont pas été intégrés au montage final. On peut imaginer que Pastrone les avaient spécifiquement conçus comme une alternative à la composition spatiale en profondeur, dont l'une des caractéristiques est, au contraire de ladite figure, de préserver l'intégrité de l'espace représenté.

On peut ainsi citer au moins trois exemples de pareils cas. Le premier que nous convoquerons se trouve au troisième épisode, au moment de l'action où Sophonisbe reçoit un cadeau de la part de Massinissa. Dans les chutes, nous trouvons deux prises à peu près semblables, qui représentent la totalité de la scène, tournée en continuité (en plus d'une troisième qui ne comporte que le début de cette action). Sophonisbe, dans sa chambre, se dirige vers la fenêtre; une esclave la rejoint et lui tend un coffret, don de Massinissa; Sophonisbe ordonne alors aux servantes de s'éloigner et s'assoit pour contempler le présent qui lui a été 
offert. On trouve aussi un autre plan (semblable à la dernière partie de l'action tournée en continuité) dans lequel Sophonisbe est assise, en plan moyen, occupée à contempler le contenu du coffret. Puis, deux prises d'un plan de Sophonisbe, debout devant la fenêtre, qui pourrait être inséré comme raccord dans l'axe, à l'intérieur du plan où on la voit qui se tient à la fenêtre. Dans le montage final, le film est organisé de manière différente, puisque la continuité de l'action est découpée en plusieurs plans, mais on n'y trouve aucune trace du raccord dans l'axe sur Sophonisbe, debout et seule devant la fenêtre. L'action y est carrément "morcelée", d'abord par un montage qui alterne entre la chambre de Sophonisbe et la salle du palais royal de Carthage où l'esclave se rend pour recevoir de Massinissa le don à porter à sa patronne; puis, par un intertitre et un raccord dans l'axe, totalement absent du matériel exclu, à la faveur duquel Sophonisbe, debout devant la fenêtre avec son esclave, reçoit des mains de celle-ci le cadeau de Massinissa; donc, par le truchement d'un raccord dans l'axe plus explicatif que contemplatif qui, comme toujours, garde ses distances et s'approche juste assez pour permettre de mieux voir l'essentiel de l'action.

On trouve un deuxième exemple de cela au cinquième épisode, alors que Fulvio escalade les murs de la ville de Catana en grimpant sur une pyramide de soldats tenant leurs boucliers au-dessus de leur tête. Il y a, dans le film en version finale, une progression en trois plans, qui fait passer le point de vue d'un plan d'ensemble, montrant les murs et la pyramide de boucliers, à un plan moyen, nous rapprochant de l'action. On trouve cependant, dans les chutes, un plan qui représente un rapprochement ultérieur de la caméra vers le coeur même de l'action décrite par cette scène (Fulvio arrive au sommet du mur et passe de l'autre côté), qui aurait pu être monté comme raccord dans l'axe, mais qui a été éliminé.

Un troisième exemple, le plus intéressant peut-être, intervient au cours du second épisode, quand le grand prêtre choisit les fillettes qui seront offertes en sacrifice à Moloch. L'analyse du matériel exclu nous permet de déduire que l'action avait été initialement conçue en deux parties. Une première partie, plutôt descriptive, campant le lieu dans lequel se déroule l'action et à 
l'intérieur duquel un carrello latéral vers la gauche rejoint Croessa et Cabiria au moment même où le grand prêtre s'approche d'elles. Et une deuxième, pour ainsi dire explicative, susceptible d'être insérée dans la première, et dans laquelle le grand prêtre, dans un plan plus rapproché, choisit certains enfants (photo $n^{\circ} 11$ ). Ce plan n'a pas été intégré dans le montage final, qui montre l'action en continuité, sans coupes ni rapprochements explicatifs d'aucune sorte.

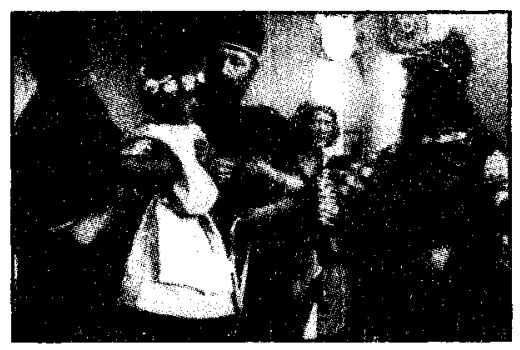

Photo $\mathrm{n}^{\circ} 11$

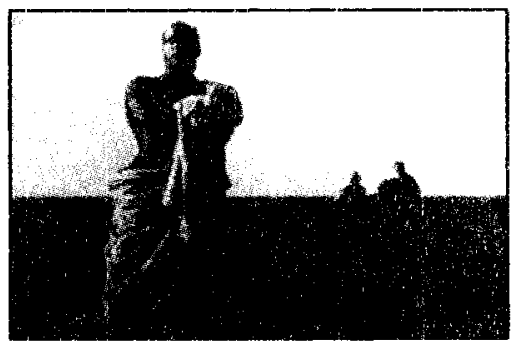

Photo $\mathrm{n}^{\circ} 12$

Cabiria (Giovanni Pastrone, 1914)

Ce troisième exemple est intéressant non seulement parce qu'il montre bien que, pour Pastrone, le maintien de la continuité spatiale est vraiment important et que, quand il le peut, il évite d'effectuer des coupes, mais il est intéressant aussi parce qu'il met en évidence le soin pris par lui dans la composition spatiale en profondeur. En effet, le plan que Pastrone n'a pas intégré, bien qu'il soit assez rapproché de la scène, montre une action qui se produit sur deux plans de l'image: au premier plan, le grand prêtre choisit une fillette, tandis qu'à l'arrièreplan, Croessa observe la scène avec inquiétude, comprenant le danger que court Cabiria.

Ce n'est donc, on l'a vu, qu'avec beaucoup de parcimonie que l'on a recours, dans ce film, à la fragmentation de l'espace (par le biais de raccords dans l'axe ou de changements d'angles de prise de vues - et dans les cas seulement où les circonstances le justifient). La situation est cependant tout autre en ce qui concerne le maintien de l'intégrité spatiale par le truchement de la composition en profondeur. Dans Cabiria, la composition 
spatiale en profondeur semble en effet être la préoccupation principale de toute la mise en scène, puisque c'est cette composition qui permet de représenter l'espace dans son intégrité, en faisant émerger sa composante tridimensionnelle. A l'instar du carrello et à l'instar des déplacements des acteurs - à qui l'on fait traverser le champ selon des trajectoires susceptibles de mettre en valeur la troisième dimension - , la composition spatiale en profondeur offre une alternative à la mobilité spatiale obtenue par la succession de plans plus ou moins rapprochés et l'adoption de divers angles de prise de vues sur l'action représentée.

Dans Cabiria, presque tous les plans en intérieur sont composés en profondeur, notamment grâce aux décors construits, qui sont conçus de manière à accentuer le déploiement de l'espace sur plusieurs niveaux. Dans ces décors, les acteurs sont souvent soigneusement disposés en arrière-plan, de manière à attirer l'attention du spectateur sur la profondeur du champ; ou alors, ils se tiennent au premier plan, prêts à traverser le champ en traçant des trajectoires qui donneront du volume à l'image. C'est la même situation qui prévaut dans les extérieurs, où les décors sont également conçus en fonction du déploiement eni profondeur. Les tournages en extérieur permettent même souvent de créer des effets supplémentaires, comme l'amplification du contraste entre la dimension de personnages postés au premier plan et celle de ceux qui évoluent en arrière-plan. Plutôt que d'opérer sur la variation des points de vue, on privilégie, dans Cabiria, une mise en cadre qui met en relief les différents niveaux de profondeur. Ainsi, dans ce film, l'analyse de l'espace semble-t-elle obéir à une sorte d'obsession eu égard à la troisième dimension, qui se traduit par l'élaboration d'une série de figures de composition en profondeur, selon la typologie suivante:

\section{- COMPOSITION EN DIAGONALE}

Il s'agit du cas le plus courant (notamment en raison de la fréquence du recours au carrello). L'exemple que nous avons choisi pour en illustrer le fonctionnement est aussi l'un des plus intéressants par rapport à ce corpus "auxiliaire" que repré- 
sentent les chutes: il s'agit du plan dans lequel Maciste et son patron Fulvio Axilla apparaissent pour la première fois dans le film. Un intertitre annonce la chose: "Fulvio Axilla, patricien romain, en compagnie de son esclave Maciste, vit incognito à Carthage, surveillant secrètement les mouvements de la République rivale." Le plan qui montre les deux héros du film renverse la hiérarchie implicite du texte écrit, puisqu'il met en relief de façon décisive le corps de Maciste, par l'intermédiaire, justement, d'une "composition en diagonale", qui s'étale dans la profondeur. Le cadrage et la mise en scène accentuent en effet le contraste entre le corps imposant de Maciste, situé au premier plan, à gauche, et le corps de Fulvio, plus menu, situé à droite, en arrière-plan (photo $n^{\circ} 12$ ). Pareille composition en diagonale permet de réunir dans un même cadre l'équivalent d'un plan moyen (Maciste) et d'un plan de demi-ensemble (Fulvio, occupé à saluer un ami qui s'en va), de façon à partager l'attention du spectateur entre l'un et l'autre.

Nous disposons, pour ce plan, de six prises rejetées. Cinq d'entre elles ont été tournées sur le même site: une jetée, au bord de la mer. La sixième a été tournée devant des rochers. C'est ce site quì a finalement été retenu. Dans chacune des six prises rejetées, toutefois, on retrouve le même type de composition, avec quelques variations quant à la valeur relative entre les deux corps. Maciste, immense, occupe toujours la partie avant du champ, tandis que Fulvio, décidément plus petit, occupe toujours l'arrière-plan, jusqu'à ce qu'il s'approche de l'homme fort, en traversant le champ selon un tracé en diagonale, et qu'il retrouve une dimension proportionnelle à celle de son compagnon. Une fois instauré, le contraste initial entre les deux corps ne sera jamais rectifié, nulle part dans le film.

\section{- COMPOSITION SINUSOÏDALE}

Cette appellation est empruntée à Pastrone lui-même ". Le plan fixe qui exemplifie le mieux ce type de composition est sans aucun doute celui qui représente Hannibal au milieu des Alpes, en compagnie de deux soldats. Parmi les chutes, il existe deux prises de ce plan, qui sont particulièrement éclairantes. La premièr correspond à ce que l'on appellerait, aujourd'hui, un plan 
américain, pour lequel la caméra a dû être placée à proximité du personnage: Hannibal apparaît seul, cadré aux genoux, entouré des sommets alpins, qui sont ainsi relégués au rôle de toile de fond, et qui, par le fait même, semblent dominés par le corps imposant du général carthaginois (photo $n^{\circ} 13$ ). Il s'agit d'un plan bien réussi, mais carrément intime. Sans être un gros plan, il est toutefois tourné suffisamment à proximité du personnage pour s'introduire dans son espace intime et donner au spectateur l'illusion d'avoir accès à ses pensées les plus secrètes et faire de lui l'unique point d'intérêt, presque en relief par rapport à un paysage à peine perceptible. Ce plan n'a pas été conservé dans le montage final ${ }^{12}$. Une seconde prise présente un cadrage du même type que celui du plan en composition en diagonale que nous avons décrit plus haut - et qui montre pour la première fois Maciste et Fulvio. Hannibal y est situé au centre d'une hypothétique trajectoire diagonale, entre deux soldats postés de manière à paraître plus bas que lui (photo $\mathrm{n}^{\circ} 14$ ). Même si elle peut nous sembler particulièrement réussie, cette prise a aussi été rejetée, au profit d'un plan plus composite, dans lequel le corps d'Hannibal domine de façon encore plus nette les deux soldats, visibles en partie seulement et privés ainsi de toute identité (ils en avaient une, au contraire, dans la prise éliminée). Dans le plan conservé, Hannibal se trouve au centre d'une hypothétique trajectoire sinusoïdale, dont les deux extrémités sont représentées par les corps à peine visibles des deux soldats. Ceux-ci, en tant qu' "accessoires", tracent les lignes d'un parcours oblique, de type sinusoïdal justement, qui accentue la

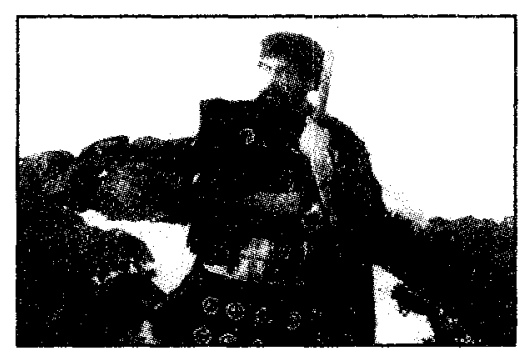

Photo $\mathrm{n}^{\circ} 13$

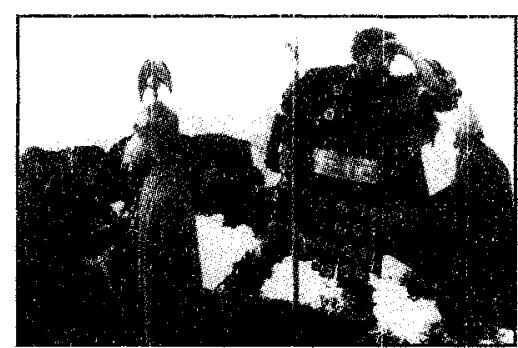

Photo $\mathrm{n}^{\circ} 14$

Cabiria (Giovanni Pastrone, 1914) 
profondeur de l'espace, de même que le volume de la troisième dimension, bien que le corps d'Hannibal, toujours plus grand, en domine le centre (photo ${ }^{\circ} 15$ ).

\section{- COMPOSITION À LA VERTICALE}

On trouve un cas intéressant de ce type de composition au troisième épiscide, dans la scène où Maciste s'enfuit dans les jardins du palais de Carthage, la petite Cabiria dans les bras. Maciste se cache entre les cactus avant de rejoindre Sophonisbe pour lui confier la fillette. Il s'agit d'une scène composée d'un seul plan, dans lequel Maciste, d'abord au fond, s'avance jusqu'au premier plan. La prise de vues est frontale, mais la caméra est placée de manière à permettre le déploiement de la profondeur sur la verticale, du bas de l'écran vers le haut. L'effet de profondeur est accru par la trajectoire verticale que suit Maciste lorsqu'il part du fond de l'image et s'avance jusqu'au buisson de cactus, situé au premier plan, derrière lequel il s'accroupit. C'est à ce moment que ses poursuivants entrent dans le champ, apparaissant par le bord supérieur du cadre. Ceux-ci restent dans le fond, minuscules, jusqu'au moment où Maciste, toujours accroupi, sort du champ par la droite, emportant Cabiria avec lui (photo $n^{\circ} 16$ ). Naturellement, cette composition à la verticale est accentuée par le contraste entre un Maciste "proche" et, donc, gigantesque, et des poursuivants "au loin", et, donc, minuscules; un effet qui est voulu, si l'on se fie aux chutes. En effet, il existe trois autres prises de ce plan, toutes beaucoup plus longues que celle qui fut retenue, et dans

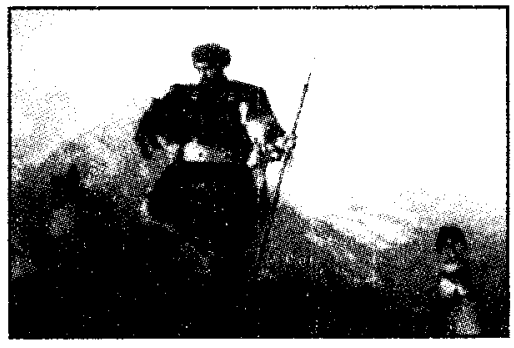

Photo $\mathbf{n}^{\circ} 15$

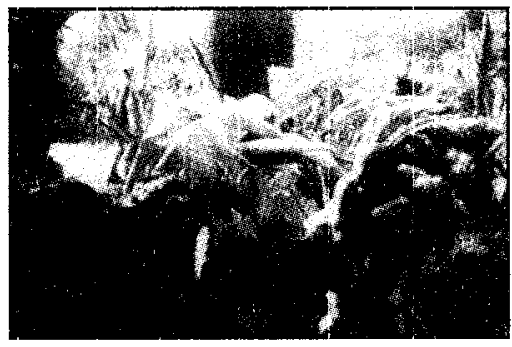

Photo ${ }^{\circ} 16$

Cabiria (Giovanni Pastrone, 1914) 
lesquelles les poursuivants n'entrent dans le champ qu'après que Maciste et Cabiria en soient déjà sortis. C'est donc dire que, dans aucune des prises rejetées, Maciste et ses poursuivants ne se retrouvent en même temps dans le champ, alors que dans la prise choisie, c'est précisément la coprésence de Maciste et de ses poursuivants qui permet d'accentuer le contraste entre les dimensions des corps, respectivement placés au premier plan et en arrière-plan, soulignant ainsi la profondeur et la verticalité de la composition.

\section{— COMPOSITION À L'HORIZONTALE}

Figure par excellence de la latéralité, l'horizontalité privilégie d'abord et avant tout la planéité, et c'est pourquoi elle est normalement incompatible avec une accentuation de la profondeur. Dans Cabiria, au contraire, grâce à Segundo de Chomòn qui, par son habileté, a rendu techniquement possible la composition très soignée de certains plans, en superposant même plusieurs prises de vues, les lignes horizontales sont distribuées les unes "derrière" les autres, de manière à accentuer la profondeur de l'espace qui les sépare l'une de l'autre. On trouve un exemple très représentatif de cette figure au cinquième épisode, alors que Maciste voit de loin le reflet du feu monter depuis le camp de Siface, où un incendie fait rage (photo $\mathrm{n}^{\circ} 17$ ).

La lumière du feu se répand horizontalement dans le fond du décor, colorant de rouge une ligne de nuages; au premier plan, par contre, Maciste observe la couleur du feu depuis un gros rocher qui dessine une ligne horizontale, elle-même symétrique

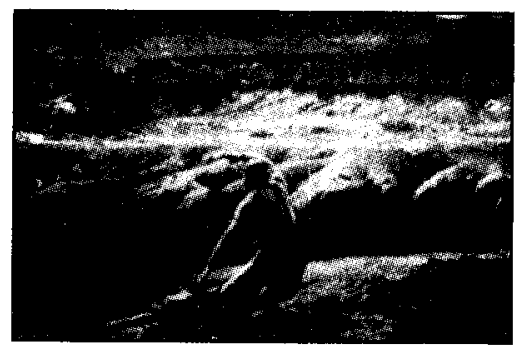

Photo n" 17

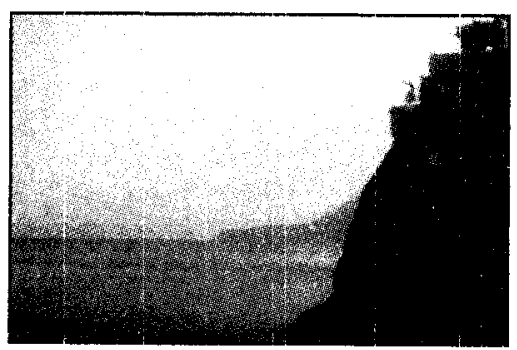

Photo ${ }^{\circ} 18$

Cabiria (Giovanni Pastrone, 1914) 
à quelques-unes des lignes du paysage. Ce plan a donné lieu au tournage de quelques prises, absolument identiques dans leur conception et leur réalisation, qui n'ont pas été retenues. Le plan choisi est celui dans lequel la ligne des nuages est la plus symétrique par rapport à celles du paysage, permettant ainsi d'accentuer l'effet de profondeur et le volume de l'espace qui sépare Maciste du feu.

Ces quatre modèles de composition spatiale n'existent pas uniquement à l'état pur dans Cabiria. Au contraire, il nous serait loisible de trouver de nombreux exemples de ce qu'on pourrait appeler des "cas mixtes", dans lesquels la composition en diagonale se mêle à une composition à l'horizontale, à la verticale ou sinusoïdale. D'ailleurs, ces distinctions que nous formulons entre les différents types de composition ne sont pas là pour le seul plaisir de proposer un modèle. Les éléments compositionnels dont notre typologie veut faire état relèvent, croyons-nous, d'une conception esthétique particulière, qui se traduit, chez Pastrone, dans des choix d'ordre "langagier».

Cetre esthétique suppose, tout d'abord, que l'on évite systématiquement le gros plan. Non pas le gros plan dit de détail (dont on n'a, par ailleurs, que très peu d'exemples dans le film, comme on l'a vu), mais plutôt le gros plan du visage humain, une figure qui revient pourtant assez souvent chez certains réalisateurs américains de l'époque. Un pareil choix esthétique présente l'avantage de toujours laisser le personnage dans le contexte de l'espace qui l'abrite. Le gros plan, on le sait, a tendance à extraire le personnage de son contexte spatial et de placer le spectateur dans une relation intime avec lui, en mettant l'accent sur sa subjectivité. Dans Cabiria, les personnages ne sont ainsi jamais entièrement isolés de l'espace qui les entoure, même lorsqu'un carrello ou un raccord dans l'axe nous rapproche d'eux. C'est la raison pour laquelle le spectateur de Cabiria n'a jamais l'impression que le personnage est prédominant par rapport à l'espace dans lequel il s'insère, un effet pourtant caractéristique du cinéma contemporain de Griffith, et successivement de tout le cinéma classique hollywoodien. Cabiria met certes en scène des personnages fondés sur une psychologie et aux motivations bien définies (l'attente de 
Sophonisbe, la dévotion de Croessa, l'orgueil de Massinissa...), mais l'attention du spectateur est plutôt dirigée vers l'espace dans lequel se déroule le drame: le temple de Moloch, les rues de Carthage, les Alpes... La chose est également due au fait que, quand Pastrone rapproche le spectateur d'un personnage, il le tient toujours en quelque sorte à distance; il lui permet de l'observer, mais jamais de franchir le seuil de son "intimité ».

En contrepartie, si le gros plan de visage est absent, il y a abondance de plans moyens et de plans d'ensemble. Ceux-ci permettent en effet de bien situer les personnages dans l'espace et ils se prêtent bien à la composition spatiale en profondeur. Ils permettent aussi le respect de l'intégrité spatiale, qui constitue le véritable moteur du projet esthétique à la base de Cabiria. Limitons-nous à ne citer qu'un seul exemple, ce sera d'ailleurs le dernier, qui survient au troisième épisode quand, fuyant Carthage, Fulvio se jette dans la mer (photo $n^{\circ 18)}$. Encore une fois, nous sommes devant un cadre très élaboré, bien que sans profondeur aucune. Cette mise en à-plat n'empêche toutefois pas d'embrasser dans le champ la totalité des éléments importants pour l'action: les rochers à pic, la mer, le profil des personnages, le plongeon de Fulvio qui remonte tout de suite à la surface en nageant rapidement... Pour ce plan, nous disposons encore une fois de nombreuses prises rejetées, qui relèvent toutes de la même conception et présentent toutes la même composition.

Ce n'est pas tout. La conception esthétique fondée sur une certaine obsession de la profondeur, qui est implicitement à l'œuvre dans Cabiria, explique non seulement le privilège accordé, sur le plan "langagier", à cette partie restreinte de l'échelle des plans qui va du plan moyen au plan d'ensemble, mais c'est elle qui fait aussi en sorte que l'on privilégie deux types, rares, de mouvements: celui, très particulier, des personnages (cf. supra) et, surtout, celui de la caméra sur son carrello, dans des déplacements qui favorisent la mobilité de l'espace scénique comme alternative à la mobilité du point de vue, et qui guident, ainsi, le regard du spectateur le long de trajectoires internes au champ, dans un décor disposé de façon songée. Dans Cabiria, en effet, on trouve non seulement toujours 
plusieurs niveaux, coexistants, de profondeur, mais encore y a-t-il, toujours, un flux de communication entre eux. Soit que le personnage traverse le champ en passant constamment d'un niveau de profondeur à l'autre, soit que la caméra circule, grâce au carrello, d'un niveau à l'autre, permettant ainsi que l'on guide l'attention du spectateur sans avoir recours à la fragmentation de l'espace et au changement de plan.

Ces trois paramètres langagiers que sont la mobilité interne (comme alternarive à la mobilité du point de vue), la composition en profondeur (qui implique par nécessité un respect de l'intégrité spatiale) et la distribution de l'action sur plusieurs niveaux qui communiquent entre eux réalisent, dans Cabiria, un idéal de représentation tridimensionnelle, qui laisse le spectateur dans les marges de l'œuvre qu'il consomme. C'est dire qu'ils servent à instituer une spatialité tridimensionnelle "à circularité limitée " qui, tout en lui permettant de se rapprocher de la scène en deçà de certaines limites, ne situent jamais, ou presque, l'instance spectatorielle au centre même de l'action. Le processus de "centrement" constitutif du mode de représentation que le cinéma américain est alors en train de mettre en place ${ }^{13} n^{3} y$ est, de cette manière, pas mis en branle. À tel point que, comme on l'a vu, malgré la présence de nombreuses figures de montage, l'utilisation de celles d'entre elles qui contribuent à faire de l'espace filmique un espace "circulaire" sont, dans Cabiria, réduites au minimum (l'une des seules exceptions consistant dans cette scène de la rencontre entre Croessa, Fulvio et Maciste, qui se fait à la faveur d'un retournement de $180^{\circ}$ ). $\grave{A}$ tel point aussi que l'usage même du carrello est, dans Cabiria, un expédient qui, plutôt que d'impliquer le spectateur, le tient "en marge" de l'action représentée, en quelque sorte extérieur à elle. Somme toute, il faut convenir de ce que le spectateur de Cabiria n'est, finalement, jamais libre de circuler à son goût dans cet espace pourtant articulé de la fiction que nous présente le film, même quand un "carrello-avant" le rapproche effectivement du centre de l'action ni même quand c'est un raccord dans l'axe qui produic le même effer.

Une telle représentation tridimensionnelle de l'espace scénique n'est toutefois pas le fait du seul Cabiria. Bien que, sous 
certains aspects, le film de Pastrone soit un film "extrême", il n'est pas pour autant un cas isolé. L'étude de ce film nous éclaire sur un paradigme qui est en train de s'esquisser à cette époque - la première moitié des années 1910. Ce paradigme oppose un style cinématographique, concentré surtout aux États-Unis, privilégiant l'analyse de l'espace à travers sa fragmentation et mettant la narrativité au poste de commande, et un autre style qui se concentre pour sa part principalement en Europe, et qui privilégie, au contraire, l'analyse de l'espace à travers sa composition en profondeur, dans un but qui, tout en restant également narratif, ne fait pas de la narrativité le critère absolu. C'est dans cette perspective que Cabiria n'est pas seulement un film dans lequel la composition spatiale en profondeur prend nettement le pas sur une analyse spatiale opérée par le type de montage que Griffith, par exemple, développe dans les films qu'il a réalisés après avoir quitté la Biograph (comme dans The Avenging Conscience, 1914; Home Sweet Home, 1914; ou The Birth of a Nation, 1915). C'est aussi un film se situant au cour d'une tradition plus proprement européenne, qui privilégie la composition spatiale en profondeur ainsi que la recherche d'une mobilité de l'espace scénique différente de celle que l'on obtient par le changement de plan.

Traduit de l'italien par Stéphanie Côté, en collaboration avec E. Dagrada et $A$. Gaudreault

Université de Bordeaux 3, Université de Montréal et University of Chicago

\section{NOTES}

1 Ce rexte est déjà paru en icalien dans une version plus longuce, sous le titre «Lo spazio mobile. Del montaggio e del carrello in Cabiria ", dans Paolo Bertetto e Gianni Rondolino (a cura di), Cabirin e il suo tempo, Torino/Milano, Musco Nazionale del Cinema/Editrice Il Castoro (1998), p. 150-183. Nous remercions les éditeurs d'avoir permis la parution de sa version française. la partie du texte original non intégrée dans le présent arricle fera l'objet d'une parution ultérieure dans CiNéMAS.

2 Cette recherche a été réalisée en collaboration avec le GRAFICS (Groupe de recherche sur l'avènement et la formation des institutions cinématographique et 
scénique) de l'Université de Montréal, subventionné par le Conseil de recherches en sciences humaines du Canada et par le Fonds FCAR du Québec. La contriburion d'André Gaudreault a été réalisée dans le cadre d'une recherche sur l'avènement et le développement du montage, rendue possible grâce à une bourse Killam (1997-1999) attribuée par le Conseil des Arts du Canada. Les auteurs remercient Stéphanie Côté, du GRAFICS, pour son support technique; Paolo Bertetto, du Museo Nazionale del Cinema de Turin, pour sa généreuse disponibilité; Claudia Gianetto et Alessandra Torrione, du Museo Nazionale del Cinema de Turin, pour leur diligence.

3 La restauration de Cabiria, réalisée en 1995 par le Museo Nazionale del Cinema, avec la contribution du "Projer Lumière", a ramené le film de Pastrone à une longueur avoisinant celle de la copie présentée le 18 avril 1914 au Teatro Vittorio Emanuele de Turin. Grâce à 75 ajouts, la version restaurée atteint maintenant une longueur de 3343 mètres, alors que la copie la plus complète disponible dans la version de 1931 ne comptait que 3132 mèrres (le visa de censure original, conservé aux Archives papier du Museo, indique une longueur de 3364 mètres).

4 Une partie de ce règlement, antérieur à 1914 (cf. Museo Nazionale del Cinema de Turin, Notiziario 40/41, 1981-1982, document n"17: p. 17-22), est reproduite dans Paolo Cherchi Usai, Giovanni Pastrone, Firenze (La Nuova Italia, 1985). La référence à l'interdiction de tourner à nouveau une scène si cela n'est pas «absolument nécessaire" est, pour sa part, rapportée par Sergio Toffetti dans "Pastrone a Torino, ovvero l'opera lirica all'epoca dell'automobilen, dans Sergio Toffetti (direction), Il restauro di Cabiria (Torino, Lindau/Museo Nazionale del Cinema, 1995).

5 Pour la distinction entre "profilmique" et "filmographique", voir André Gaudreaulı, Du littémire au filmique. Système du récit (Paris/Québec, Armand Colin/ Nota bene, 1999).

6. On trouve un exemple de ceci dans les plans conçus pour représenter la première rencontre entre Croessa, Fulvio et Maciste. Dans le matériel exclu, nous disposons d'un plan qui représente une sorte de contrechamp de l'un des plans retenus, mais dans lequel Maciste occupe, contre toute attente, la même partie du cadre que le plan qui montre l'action qui le précède, logiquement. Il s'agit là d'une "erreur" qui a peutêtre motivé l'exclusion du plan en question, quoique son tournage peut aussi tenir d'une volonté de faire l'essai d'une autre perspective, d'un autre point de vue, afin de se laisser l'option d'un dispositif différent. On trouve aussi un bel exemple d'une conception iconographique alternative dans la scène du rêve de Sophonisbe, qui a été tournée à deux reprises. La prise exclue est assez similaire à une autre scène "onirique" réalisée auparavant par de Chomón chex Parhé, en 1905, pour le film La Poule aux azufs d'or. Voir à ce sujet, Elena Dagrada, "La morale de la fable (ou de quelque Poule aux oufs d'or chez Pathé)", dans Jean A. Gili, Michele Lagny, Michel Marie, Vincent Pinel (direction), Les Vingt Premières Années da cinéma franf̧ais (Paris: Presses de la Sorbonne Nouvelle, 1995).

7 Une analyse comparative entre le film Cabiria et ses chutes en rapport avec les problèmes de temporalité pourrait être fort intéressante. Limirons-nous, pour l'instant, à dire que les prises sélectionnées pour faire partie du montage final sont toujours d'une duréce inférieure à celle des prises rejetées, pas nécessairement parce l'on a coupé les extrémités des prises retenues, mais parce qu'il s'agit de prises dans lesquelles l'action se déroule plus rapidement, de façon à permettre, peut-être, la coprésence dans le champ de plusieurs éléments de l'action (cf. plus avant l'analyse de ce que nous avons nommé la " composition verticale").

8 À propos de ce conccpt, voir surtout: Ben Brewster, "La mise en scène en profondeur dans les films français de 1900 à 1914 ", dans Pierre Guibbert (direction), Les I'remiers Ans du cincéna frangalis (Perpignan: Institut Jean Vigo, 1985); Barry Salt, Film Stvle and Technology: History and Analysis (London: Starword, 1983, p. 113-116); 
Tom Gunning, "Nores and Qucries About the Year 1913 and Film Style: National Styles and Deep Sraging", 1895, numéro hors série consacré à L'Année 1913 en France, 1993. Voir également Ben Brewster er Lea Jacobs, Theater to Cinema. Stage Pictorialism and the Early Feature Film (Oxford: Oxford University Press, 1997); David Bordwell, en particulier On the History of Film Style (Cambridge: Harvard University Press, 1997); et Yuri Tsivian (dans une conférence inédite sur le cinéma russe des années dix, prononcée à Bradford en 1995, au colloque Celebrating 1895). Voir aussi, de Tsivian, le cédérom Immaterial Bodies: Cultural Anatomy of Early Russian Films (Los Angeles, USC Electronic Press, 1999), qui comprend une section sur Cabiria À propos de Cabiria, voir également Antonia Lant, "Haptical Cinema", October 74, 1995, p. 44-73.

9 Cas de figure que l'on associe souvent au champ/contrechamp mais qui, stricto sensu, n'en est pas un. Nous avons opté, ici, pour une utilisation restreinte de l'expression, et avons limité son usage pour la description des seuls cas impliquant des échanges entre personnages.)

10 Selon nous, la théorie du cinéma ne fait pas suffisamment la distinction entre "gros plan de visage" et "gros plan d'objet". Pourtant la distinction existe bel et bien dans la tradition, qui suggère de parter de "gros plan ", pour le gros plan de visage, et de "détail " pour le gros plan d'un objet ou de la partie d'un corps. Sur la nécessité de distinguer entre "gros plan" et "détail", voir Elena Dagrada, "La lente della nonna. Una scuola per lo sguardo", dans Riccardo Redi (direction), Il primo cinema inglese (Roma: Di Giacomo, 1990); et La rappresentazione dello sguardo nel cinema delle origini in Eurropa (Bologna: Clueb, 1998).

11 Voir les déclarations de Pastrone dans Georges Sadoul, "La tecnica rivoluzionaria nella Cabiria di Pastrone". Cinema, nuova serie, anno IV, n" 58, 1951; et Mario Verdone, "Pastrone, ulcimo incontro", Bianco e Nero, anno XXII, n" l, gennaio 1961.

12 On peut même faire l'hypothèse qu'il avait été conçu pour permettre un raccord dans l'axe, qui aurait été monté à la suite du plan, plus large, qui est resté dans le montage définitif mais, le cas échéant, il aurait été rejeté pour les mêmes raisons.

13 Noël Burch, La Lucame de l'infini. Naissance du langage cinématographique (Paris: Nathan, 1990). À propos de la profondeur de champ dans le cinéma européen précédant la diffusion du "centrement "hollywoodien, voir aussi, toujours de Noël Burch, "Germinal avant le sujet ubiquitaire", 1895 (numéro hors série consacré à L'Année 1913 en France), octobre 1993.

\section{OUVRAGE CI'TÉ}

Bordwell, David. "La Nouvelle Mission de Feuillade; or, What Was Mise-en-Scène?". The Velvet Light Trap, n" 37 (1996), p. 22. 